\title{
Design of an Electromechanical Prosthetic Finger using Shape Memory Alloy Wires
}

Conference Paper · October 2017

CITATIONS

0

3 authors, including:
READS

19

\section{Louis van der Elst}

B.S. in Electrical Engineering, Pennsylvania Sta...

1 PUBLICATION 0 CITATIONS

SEE PROFILE

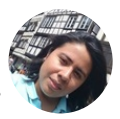

Serket Quintanar-Guzmán

University of Luxembourg

8 PUBLICATIONS 8 CITATIONS

SEE PROFILE

Some of the authors of this publication are also working on these related projects: 


\section{Design of an Electromechanical Prosthetic Finger using Shape Memory Alloy Wires}

\author{
Louis A. van der Elst \\ Research Unit in Engineering Sciences \\ University of Luxembourg \\ Luxembourg, G.-D. of Luxembourg \\ Email: louis_vanderelst@vdelst.eu
}

\author{
Serket Quintanar-Guzmán \\ Interdisciplinary Centre for Security, \\ Reliability and Trust (SnT) \\ University of Luxembourg \\ Luxembourg, G.-D. of Luxembourg
}

\author{
Prof. Jean-Régis Hadji-Minaglou \\ Research Unit in Engineering Sciences \\ University of Luxembourg \\ Luxembourg, G.-D. of Luxembourg
}

\begin{abstract}
This research concerns the design and prototyping of an artificial middle finger, using Shape Memory Alloys (SMAs), PolyLactic Acid (PLA), and other technologies. The design is a biomimicry of the human biological anatomical and muscular systems. After briefly describing the operational features and functioning of natural striated muscles, the document reviews the features, advantages and disadvantages of SMAs in the perspective of their use as an actuator of a prosthetic finger. Using different design parameters, such as the lightness of the device, actuation complexity, and resilience, a working prototype is proposed meeting the established criteria.
\end{abstract}

\section{INTRODUCTION}

Prosthetics date back to the beginning of human civilization, first mentioned in the Vedas approximately 1500 BC [17], and then first technically recorded in the late 16th century by Ambroise Paré [7]. Today, prosthetic novelty is seen through power propulsion ankles, enhancing dancing performances [8]; thus, the creation of super-human capabilities.

Commercially, prosthetics are still described as being heavy when patients use them [12] and systems such as osseointegrated attachment or adaptable sockets mechanisms are still being researched [5]. Heavy duty mechanical mecanisms are used in commercially available hands that greatly increase the weight of the design. Hence, there is a need for a solution that feels more natural to the patient.

According to the analysis by the authors in [12], the smallest large finger weight recorded was $35 \mathrm{~g}$, hence this study will use this as a design criteria.

The human hand has 22 DOFs, whereas the prosthetic Dexmart Hand, with the highest amount of DOFs, by the authors in [10] has 18 DOFs, actuated with tendon actuators.

In a study [11], different string and DC motor solutions for the finger mechanisms to achieve more DOFs are developed, based on an in-depth analysis of the muscular systems of the hand. His prototype include pulley systems, which make the design mechanically complex, and relies on motorized solutions in the hand or in the forearm to actuate the simple metal wires. SMA wires provide an alternative to that method.

The work of the author in [2] proves that with a rearrangement of a servo motor and an additional gearing system, a finger can be actuated using standard mechanical solutions within the finger. The assembly is complex and the DIP joint is fixed.
To overcome the inconveniences currently present in prosthetics, we designed a lightweight, cost-efficient, sustainable artificial solution, actuated using Shape Memory Alloy (SMA) wires. This research proposes a new set of rules to creating prosthetic hand. Additionally, we highlight the extent of developing prosthetics, through simplification and biomimicry, using new technologies. The focus of this research is deliberately confined to one finger as a proof of concept.

This paper will cover the biological and material background to this research, provide the rationale behind this author's design guidelines to obtain proper biomimicry, and describe the design creation process and evaluation of the prototype device. The remaining of the paper is organized as follows: Section II covers the biology of the finger and the systems that allow it to move; Section III looks into the properties of shape memory alloys, the material science behind it, and its current applications in prosthetics; Section IV describes the procedural steps in creating the finger prototype; and Section V discussing the observation on the actuation of the design, a look at how the design can be improved for a better performance, and an explanation on the foundation it lays out for further research opportunities.

\section{WORKING SySTEMS OF THE FINGER}

The first step to the biomimetics of a biological system, is to understand its working mechanisms. This section covers the structural and actuation systems that allow the movement of the human finger.

\section{A. Finger Anatomy and Muscles}

Except for the thumb, the finger or digitus, is composed of three different phalanges, namely the Distal (DP), Intermediate (IP), and Proximal Phalanx (PP), which are joined to the hand via the Metacarpal Bone (MB). The finger has two surfaces, on the bottom and on the top called the palmar and dorsal surface, respectively. See Figure 1, for a visual representation of the finger, as this paragraph explains its different anatomical properties. The phalanx is made of three parts: the base, the shaft and the head. Joints owe their names to the heads of the two phalanges or bones they interconnect: the Distal Interphalangeal (DIP), Proximal Interphalangeal (PIP), and the MetaCarpoPhalangeal (MCP) joints [5]. The muscles that 

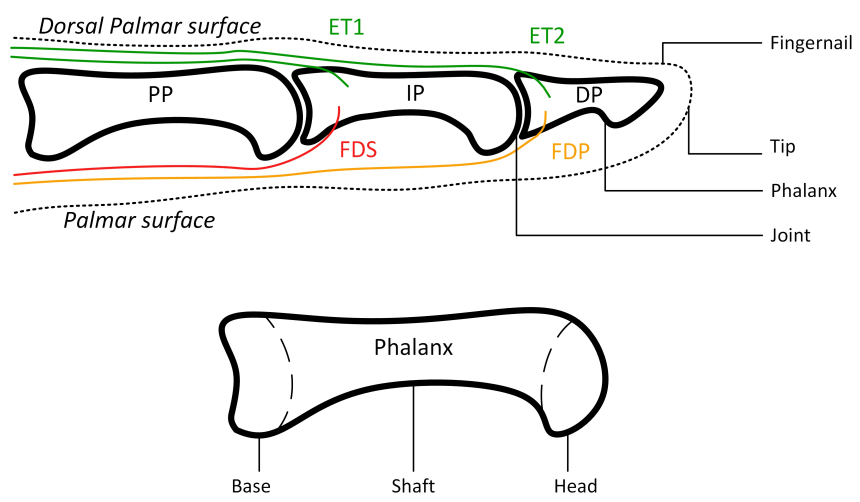

Fig. 1. Anatomy of the finger and the phalanx including the extrinsic tendons.

allow the finger to move come from the hand (intrinsic) and the forearm (extrinsic) and insert into the base of the DP or IP. Due to the nature of SMA wires discussed in Section III, this study specifically focuses on extrinsic muscles. Flexion occurs partly due to the Flexor Digitorum tendons. The tendon that reaches the IP is the Superficialis (FDS) tendon, and the DP is the Profundus (FDP) tendon. Extension occurs partly due to the Extensor Tendons. The finger radial movements, abduction and adduction, and cirular movements, circumduction, are controlled by a different set of muscles [5].

Fingers are given motion by striated or skeletal muscles, made of hundreds to thousands of filaments of myofibrils. Myofibrils are composed an intermeshed network of long and fibrous proteins which give the motion to the finger through contraction and strain movement. This contraction is similar to the behavior of SMA wires when actuated.

\section{Shape Memory Alloy as an ACtuator}

Shape Memory Alloys (SMAs) are metal compounds that show two unique properties: shape memory and pseudoelasticity [19]. In addition, they are strong corrosion resistant alloys. Although many different types of metal and non-metal composite materials exist with similar properties - usually copper-, iron-, or nickel-titanium-based - this research uses nickel-titanium alloys developped by Dynalloy [19].

\section{A. Solid State Change}

SMAs' unique properties are achieved through thermal induction causing a non-diffusive, molecular rearrangement within the material to switch from the initial martensite solid state to an austenite solid state of the material [19]. This is achieved by heating or cooling the material above or below its characteristic transformation temperatures, respectively. The transformation temperatures vary based on the SMA materials and mix ratio. These temperatures must be strictly respected. When the material is heated and transforms into austenite, the metal hardens and reduces in volume, due to the orderly rearrangement of the molecules in the material. In addition, the part will return to the initial shape it had when it was first manufactured and shaped. For example, an SMA wire that has

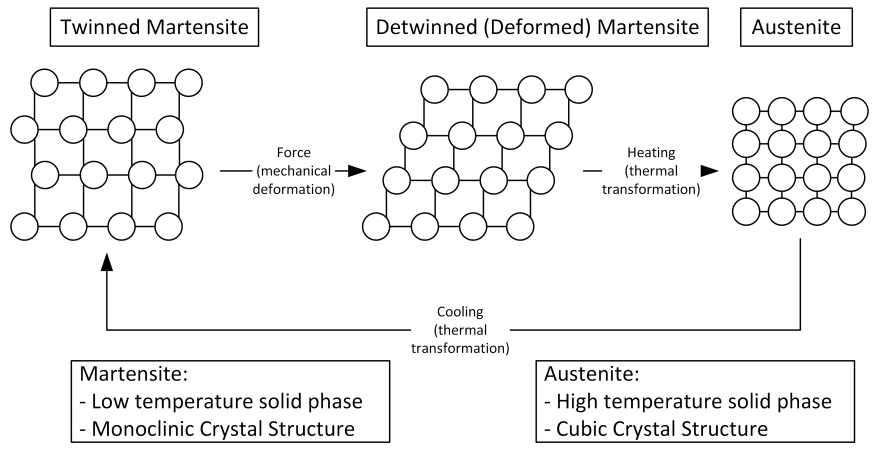

Fig. 2. Temperature-induced transformation of the crystal structure of the metal alloy.

been deformed physically, and that is then heated, will readopt its straighteness at the end of the transformation. This characteristic phenomena is called the shape memory effect. Another characteristic behaviour occurs in the martensite phase, when the material can be deformed and will recover $8 \%$ to $10 \%$ without noteable residual strain. This latter effect is called the pseudo-elastic effect [16]. Figure 2, shows the crystallographic rearrangements during the different thermal stages. Respecting the SMA's limitations, the material can be heated and cooled multiple times while keeping these properties.

\section{B. Characteristics of SMAs}

In prosthetics development, the SMA wires have advantages over other actuators such as hydraulics, pneumatics, dc/ac motors, servo motors, and other non-conventional actuators. The SMA are simple mechanisms and require no complex gearing systems. The SMA actuation is silent unlike motors, which is necessary in prosthetic development. Although the materials in themselves and the manufacturing of titanium and nickel alloys is not cheap, its availability in sheet, wire, tube or spring form makes this actuator considerably cheaper than other conventional actuators. Its miniature size allows for the most complex and space constrained actuation possible.

However, SMAs have certain disadvantages that have to be taken into account in the design. SMAs have highly non-linear behavior, hysteresis, discussed in the next section. Its actuation is of a low 4\%-6\% efficiency deformation compared to its length. It has a slow speed of response limited by its cooling and heating properties. It has little lineancy with fatigue effects outside of its recommended limits - which allows it to be reliable over millions of cyclic work - or it will be damaged and stop functioning [13].

SMA wires are a perfect means of actuation for an artificial finger. They fit in tight spaces, simplify designs, work in corrosive environments, have noiseless operation, have low costs, and are not invasive to their environment. However, their properties and limitations have to be well respected.

The behavior of SMAs is complex and difficult to predict or control. When excessive strain is applied to the material during heating, it can be permanently deformed and it adopts 


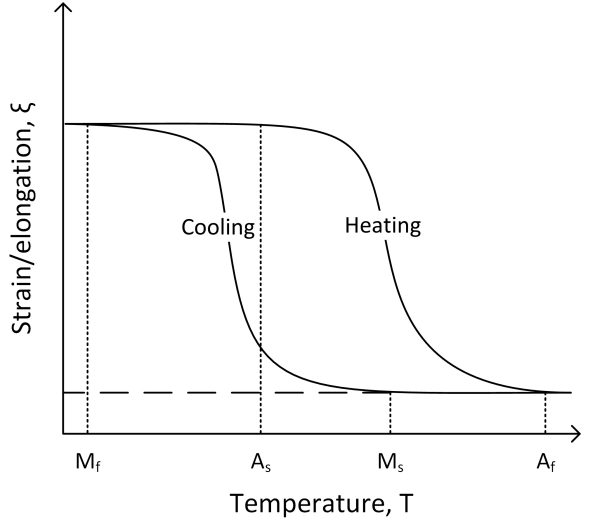

Fig. 3. The hysteresis loop.

a shape adjusted by its environment. The contraction time is directly based on the electric current input, however if the threshold is exceeded, the material is damaged and looses its shape memory and pseudoelastic properties.

The change from one solid state to another is non-linear, a behavior called hysteresis. Figure 3, shows the hysteresis in relation to the shape memory effect. "A" and "M" stand for the austenite and martensite solid states, and indices "s" anf "f" stand for start and finish, respectively. $A_{s}, A_{f}, M_{s}$, and $M_{f}$ indicate the beginning and ending key temperatures of the state transitions. Hysteresis is dependent on the material's history, meaning that depending on the temperature change and the strain on the system, the value for different variables will vary. In other words, hysteresis is a lagging period between the heating and the cooling curves, which may explain why it is not easy to develop a control algorithm or to model the SMA wire behavior.

\section{Prosthetic Applications of the SMA wires}

Due to their properties, SMAs are used in electronics, in the aerospatial industry, in the automotive industry, and more - often as a tool for control or sensoring, in valves, in locks, in mechanical current or voltage regulators, and more. The retraction of SMA wires when the metal is heated is similar to the sarcomere contracting in muscles. SMA wires can be as small as $0.1 \mathrm{~mm}$ or less in diameter, which is comparable to muscle fibers who are a little under $100 \mu \mathrm{m}$ in diameter. The filaments of myofibril are $1 \mu \mathrm{m}$ to $2 \mu \mathrm{m}$ in diameter typically [6]. The size of the diameter does not affect the deformation length. Therefore, using this technology to replace the muscles in a prosthesis is an adequate choice.

Although there are currently no commercial prosthetic limb available, extensive research has been done on SMAs and its use and prosthetic designs have already been attempted in academia [13], where the results have shown sluggish responsiveness, dependencies on a forearm section, and low forces at the finger tips as disadvantages. This design will try to solve the sluggish response by applying a strict current as recommended on very thin wires, thus reducing temperature heating and cooling time.

The response of the wire retraction once activated should be swift once the desired temperature is reached. As for the dependencies on the forearm, these are inevitable due to the small amount of deformation possible in comparison to the length of the SMA wire. However, this should not be regarded as a drawback as they are comparable to the extrinsic muscles that actuate fingers and that run all the way back to the two forearm bones, specifically at the end of the ulna and the head of the radius, just before the elbow.

The wires have a pull force of $8.9 \mathrm{~g}$ for a wire of $0.1 \mathrm{~mm}$ in diameter. Grouping wires into a bundle allows them to be stronger while maintaining the same deformation properties, which will help to get the desired force at the fingertips. The force at fingertips should be under 10N [1].

\section{PRototype CREATION}

The prototype was developped methodically based on previous research and constraints established to fulfill our goals.

\section{A. Design Rationale}

Ideally, a prosthetic hand should be designed accordingly to the characteristics of the patient's able hand. However, studies have established a set of observations for natural and prosthetic hands which can help us establish a general criterion for an adult size replacement limb. The concept of biomimicry is, through innovation and engineering, to solve today's challenges drawing inspirations from natural systems and processes. In this case, the goal is to create a prototype artificial human finger using affordable technology to replicate the muscular and tendon system that actuate the limb.

Since the main goal of this study is to prove a new design concept using modern materials, the prototype will not have lateral or circular movements; therefore, it will not include lumbrical and interosseous muscles that are responsible for these motions. The design will take the middle finger or digitus medius as an example to biomimic. In addition, the nervous system of the finger is excluded here, as it is not a concern of this research.

Based on the natural finger kinematics and working process, following the biomimicry concept, certain requirements have been established. The created components biomimic the bone structure. The metacarpal bone prototype will be the base of the structure. The solution should be noise free, as muscles do not make a sound when they actuate our body. The nomenclature from the anatomy of the human body will be used throughout this design.

Three different SMA wire diameters were compared by actuating them and observing their deformation efficiency, their response time, their electrical consumption, and their pull force. Through a series of tests it was concluded was that the thiner the wire the better as the cooling time is much reduced. The electrical consumption is proportional to the diameter size of the wire. Bundled actuation remedies the individual weak force drawback of thiner wires. Figure 4 shows the the results of the response time. The ET has 6 wires bundled together 


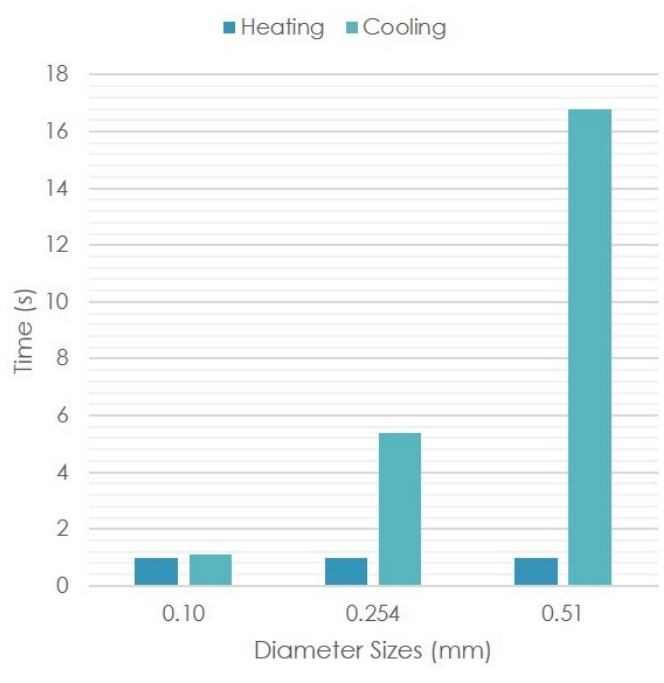

Fig. 4. Heating and cooling times of the actuation of three different SMA wires of different diameters.

and the FD has 4 wires bundled together. The pull force of the wires when heated is $143 \mathrm{~g}$ or $1.402 \mathrm{~N}$. That is, $5.611 \mathrm{~N}$ for the ET $\left(F_{E T}\right)$ and $8.417 \mathrm{~N}$ for the $\mathrm{FD}\left(F_{F D}\right)$, when the wires bundled together. The average force of a human hand at a fingertip is $6.3 \mathrm{~N}$, and prosthetic hand ranges from $9.9 \mathrm{~N}$ to $24.9 \mathrm{~N}$ [1]. In the scope of biomimetics, this bundle selection is ideal.

SMA bundles will take the place of muscle fibers in a nonequal antagonist way. Unequal meaning that the extension and flexion force are different, and antagonist meaning that sets of bundles will oppose each other in the actuation of the finger. Other systems such as springs and rubbers can be used as the antagonist partner to the SMA wire, however they wear off and are unreliable. It would require a constant maintenance of the system after the initial tuning of the SMA wires. Keeping to the recommended usage of the wires will allow them to uphold the same actuation for millions of work cycles after the initial tuning of the system.

The required current to actuate an SMA wire with a diameter of $0.1 \mathrm{~mm}$ is $0.8 \mathrm{~A}$. THe wire bundles result in a current consumption of $4.8 \mathrm{~A}$ for the ET $\left(i_{E T}\right)$ and $3.2 \mathrm{~A}$ for the FD $\left(i_{F D}\right)$. The deformation is announced by the manufacturer to be around $4.5 \%$. Predictions of the necessary length of the SMA wire extension along the forearm can be made mathematically as shown in Equation 1, where $l_{\text {reserve }}$ is the length of the SMA wires in the forearm, $l_{\text {flexed }}$ is the length of the SMA wires in the finger when flexed, and $l_{\text {extended }}$ is the length of the SMA wires in the finger when extended. The calculation results are shown in Table I.

$$
l_{\text {reserve }}=\frac{l_{\text {extended }}-(1+4.5 \%) *\left(l_{\text {flexed }}\right)}{4.5 \%}
$$

Moving on to different sections of the design, the prototype structure will be made in polactic acid (PLA) due to its light weight and its ease of printing complex parts in 3D.
TABLE I

SMA WIRE LENGTH ESTIMATIONS

\begin{tabular}{|c||c||c||c|}
\hline $\begin{array}{c}\text { SMA } \\
\text { wires }\end{array}$ & $\begin{array}{c}l_{\text {extended }} \\
\text { (in } \mathrm{mm} \text { ) }\end{array}$ & $\begin{array}{c}l_{\text {flexed }} \\
\text { (in } \mathrm{mm} \text { ) }\end{array}$ & $\begin{array}{c}l_{\text {reserve }} \\
\text { (in } \mathrm{mm} \text { ) }\end{array}$ \\
\hline FDS & 138 & 90 & 977 \\
\hline FDP & 162 & 103 & 1208 \\
\hline ET1 & 145 & 117 & 505 \\
\hline ET2 & 193 & 152 & 759 \\
\hline
\end{tabular}

TABLE II

THe REQUiREMENTS LIST FOR THE DESIGN

\begin{tabular}{|c|c|c|c|c|}
\hline N. & Requirement & Description & Priority & Difficulty \\
\hline 1 & Actuation & $\begin{array}{l}\text { SMA wires }-0.10 \mathrm{~mm} \\
\text { in diameter }\end{array}$ & High & High \\
\hline 2 & $\begin{array}{l}\text { Phalangeal } \\
\text { structure }\end{array}$ & $\begin{array}{l}\text { 3D printed polylactic } \\
\text { acid }\end{array}$ & High & High \\
\hline 3 & Joints & $\begin{array}{l}\text { Needle bearings: } 7 \mathrm{~mm} \\
\text { in diameter }\end{array}$ & High & Low \\
\hline 4 & Fixations & $\begin{array}{l}\text { Screws of } 1.0 \mathrm{~mm} \text { to } \\
1.6 \mathrm{~mm} \text { and soft ca- } \\
\text { bles soldered to copper } \\
\text { plates }\end{array}$ & High & Medium \\
\hline 5 & Control & Feedforward & Low & Medium \\
\hline 6 & Forearm & $\begin{array}{l}\text { Extension of the SMA } \\
\text { wires, electric cables, } \\
\text { and power input }\end{array}$ & High & Low \\
\hline 7 & $\begin{array}{l}\text { Flexing } \\
\text { angles }\end{array}$ & $\begin{array}{l}61^{\circ}, 48^{\circ} \text {, and } 30^{\circ} \text { for } \\
\text { the MCP, PIP, and DIP, } \\
\text { respectively [18] }\end{array}$ & Medium & Low \\
\hline
\end{tabular}

In addition, PLA is a sustainable, ecofriendly material as it is biodegradable and easy to recycle. The SMA wires will be fastened to the structure mechanically using washers, nuts, and bolts. Other solutions such as soldering do not work in this situation due to the volume reduction of the SMA wire when it heats up.

Regarding the control of the prototype, simulations of the SMA behavior is very hard especially because of its hysteresis. Therefore the control system is feedforward in this study. A combination of contact plate and strain gauge sensors would allow be a possible upgrade to provide feedback [9].

\section{B. Creation Process}

All noteworthy points are compiled into a requirements list which is the base on which the 3D modelling of the prototype will be constructed, shown in Table II.

3D modeling was done using AutoDesk Inventor 2016. The seperate components are shown in Figure 5 and the full assembly is shown in Figure 6. The full designed assembly is shown The printing was achieved by layering the parts and generating the g-code using the software Cura. An Ultimaker $2+3 \mathrm{D}$ printer was used to create the desired plastic parts. The polylactic acid (PLA) was heated at the nozzle to $210^{\circ} \mathrm{C}$ and the workplate was heated at a temperature of $50^{\circ} \mathrm{C}$ for the part to grip to the workspace. The smallest part, the distal phalanx took about 40 minutes to produce, containing $2.3 \mathrm{~g}$ 


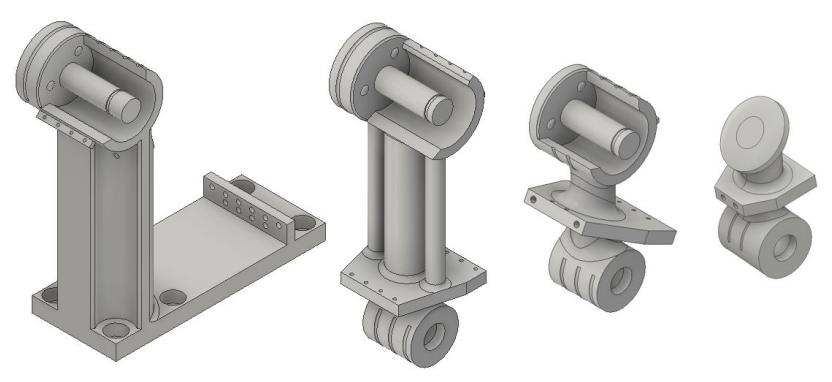

Fig. 5. 3D phalangeal and osseous components.

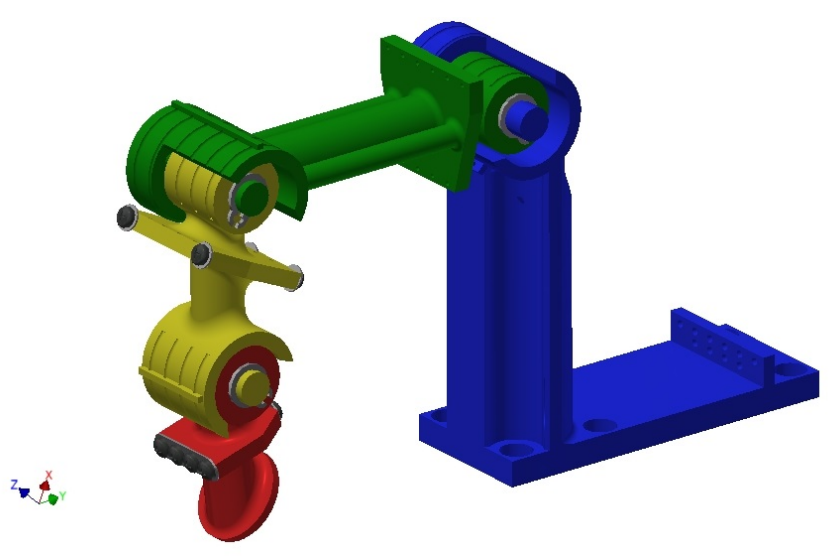

Fig. 6. 3D Model of the assembled prosthetic finger.

of material. The mecarpal took 2 hours and 47 minutes to print and weighs about $12 \mathrm{~g}$. Since the $3 \mathrm{D}$ printing time is lengthy when a large quantity of material is being used, it had to be optimized such that the least amount of material could be used, in addition to making the rigid structure as close as possible to the phalangeal structure for proper biomimicry. Using molds would be more effective in terms of finitions and series production. It was observed that for tolerances, $0.1 \mathrm{~mm}$ to $0.2 \mathrm{~mm}$ had to be added at every connection point to leave a gap for proper asssembly of the parts. At last, the design is $22 \mathrm{~mm}$ wide, is $103.97 \mathrm{~mm}$ long, and weighs $32.3 \mathrm{~g}$. The production costs are about 45 euros and the manufacturing lead time is about 14 hours.

\section{Testing the Design}

The whole system overall is $1.4 \mathrm{~m}$ in length, shown in Figure 7. Although the design works well, simple cabestan solutions can be implemented. The parts have been powered progressively to the recommended amperage as calculated. A current intensity of $600 \mathrm{~mA}$ was found to be sufficient to actuate both the ET and FD SMA wire bundles.

The printed parts, the DP, the IP, the PP, and the MB, weigh $2.3 \mathrm{~g}, 4.2 \mathrm{~g}, 6.3 \mathrm{~g}$, and $12.0 \mathrm{~g}$, respectively. The overall design (in full assembly) weighs $32.3 \mathrm{~g}$ which meets the requirement of $35 \mathrm{~g}$. Over time, each couple of wires had to be slightly readjusted, as the wires naturally adopted the forced curvatures involved in the actuation of the design. As they were repeat-

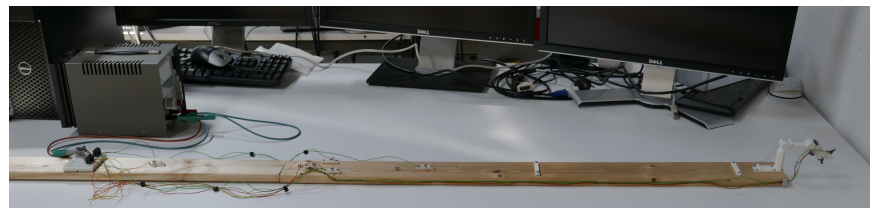

Fig. 7. Setup of the prototype forearm and finger.

edly getting heated and cooled, the sections of wires located at turning points or going through a sharp edge assumed the forced shape of those section.

For both the extension actuation, shown in Figure 8, and the flexion actuation, shown in Figure 9, the movement is achieved in two phases. The initial reaction of the bundle is about 1s, where the PP assumes its new positions at $100 \%$. In the second interval, the flexion and extension take an additional $7 \mathrm{~s}$ and $12 \mathrm{~s}$, respectively, for the IP and DP to slowly move into their positions. Moreover, the DP does not fully extend or fully flex. These deficiencies are due to the resistance of deformation of the antagonist SMA wire bundle set. The first interval reaction is fast as the other wires, having cooled, have naturally extended and offer no resistance. The second interval reaction is slow because the actuated SMA wires are elongating the antagonist wires. A solution to that would be to use electromagnets to setup a reliable bias system that temporary supplements force to fully close the finger between two specific points.

Overall the plastic pieces accommodated well with the hot wire environment. Although PLA is a recyclable cheap material, there is an opportunity to develop the design using bioplastics derived from renewable biomass sources as opposed to petroleum or natural gas plastics. In addition, in a more complex design, the proof of concept covered in this document can include the appropriate muscle or SMA structures to achieve the missing degrees of freedom that are not accounted for in other prothetic hands. A new design goal for a future project would require some research on the elasticity and the inclusion of spherical bearings for the MCPs.

\section{CONCLUSION}

We have created a new artificial finger, a reproduction of the human finger bone and phalangeal structure, actuated by shape memory alloys. This prototype is mechanically simple, is light, requires low energy consumption, is cost-friendly, uses recyclable materials, requires low maintenance, and is non-invasive to its environement. The research shows a real potential in using SMA wires to replace muscular functions, and an illustration of the versatile possibilities of additive manufacturing such as 3D printing. Additionally, a strong foundation has been laid for the development of a full hand prosthesis including: more biomimetics of the muscular and tendon system; elctromagnets to better replicate the natural finger movement; a combination of electric touching pad and strain gauge sensors; and the development of a ventilated cosmetic skin. This study provides a solution to biomedical 


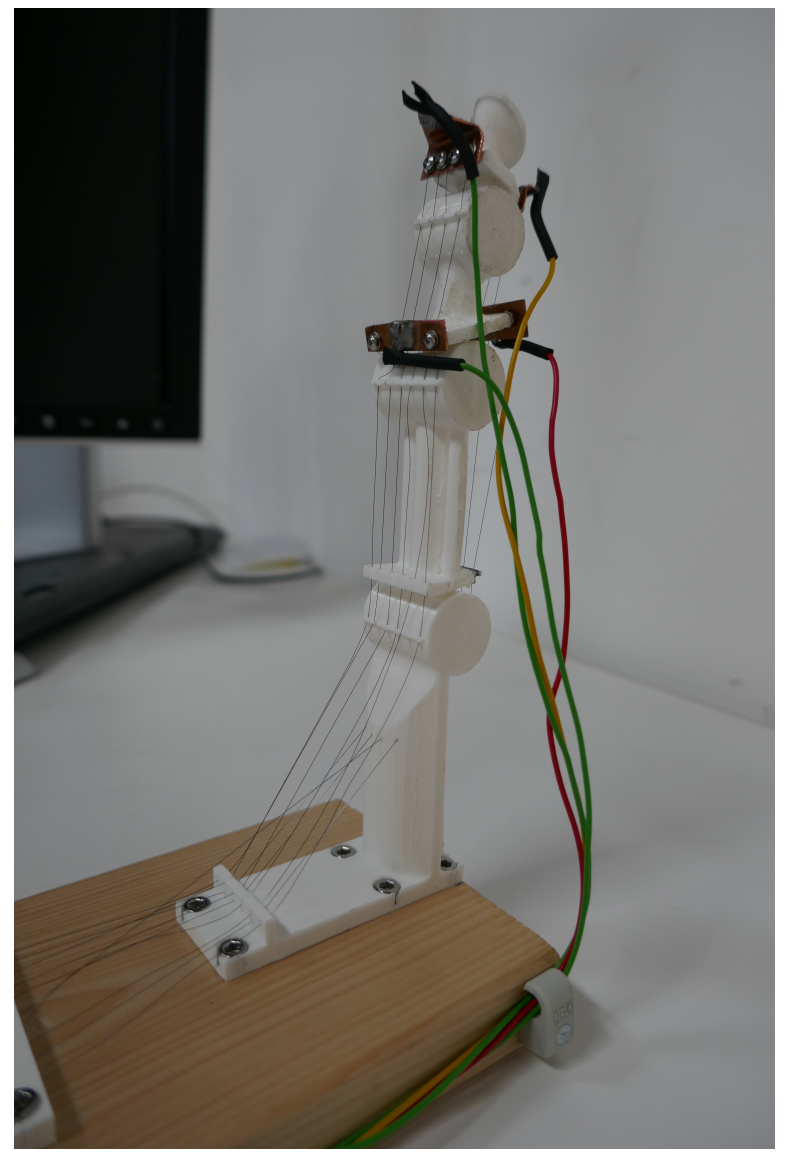

Fig. 8. Prototype extended by the SMA wire FD bundles.

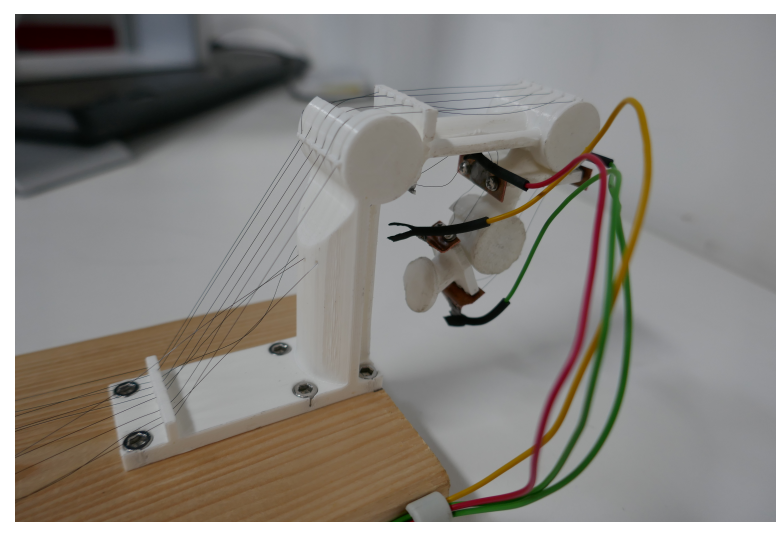

Fig. 9. Prototype flexed by the SMA wire ET bundles. engineering in developping lightweight intelligent prosthetics at affordable costs.

\section{REFERENCES}

[1] Artem Kargov, Christian Pylatiuk, Jan Martin, Stefan Schulz, and Leonhard Döderlein, A Comparison of the Grip Force Distribution in Natural Hands and in Prosthetic Hands. Disability and Rehabilitation, vol. 26, no. 12, pp. 705-711, 2004.

[2] Christophe Colas, Conception, Dévelopement et Réalisation d'un Prothèse de Main Robotisée. Travail de Fin d'Etude - Bachelor en Ingénieure Mécatronique, 2014.

[3] Chung Yan Liu and Wei Hsin Liao, A Snake Robot Using Shape Memory Alloys. IEEE International Conference on Robotics and Biomimetics, August 2004.

[4] David K. Blough, Sharon Hubbard, Lynne V. McFarland, Douglas G . Smith, Jeffrey M. Gambel, and Gayle E. Reiber, Prosthetic cost projections for servicemembers with major limb loss from Vietnam and $O I F / O E F$. Journal of Rehabilitation Research \& Development, vol. 47, no. 4, pp. 387-402, 2010.

[5] Finger Anatomy, Bones, Joints, Muscle Movements and Nerves. Health Hype, 2017.

[6] General Anatomy of Skeletal Muscle Fibers. Human Anatomy - Get Body Smart, 2016.

[7] Philipe Hernigou, Ambroise Paré IV: The Early History of Artificial Limbs. 2013.

[8] Hugh Herr, New Bionics Let Us Run, Climb and Dance. TED Talks, 2014.

[9] Hussein Rappel, Aghil Yousefi-Koma, and Hamid Basaeri, Shape Control of a Bio-Inspired Flexible Tail by a Shape Memory Alloy Actuator: An Experimental Study. Bi-annual International Conference on Experimental Solid Mechanics and Dynamics (X-Mech-2014), February 2014.

[10] Ivo M. Sousa, Ana R. Barbosa, Micael S. Couceiro, Carlos M. Figueiredo, and Nuno M. F. Ferreira, Exploiting the Development of Robotic Hands - A Survey and Comparison on Different Technologies. 2nd International Conference on Serious Games and Applications for Health - SeGAH, May 2013.

[11] Jakub Žajdlík, A Novel Construction and Mechanical Principles of FiveFingered Prosthetic Hand. International Journal for Theoretical and Applied Mechanics, vol. 16, no. 2, pp. 81-92, 2009.

[12] Joseph T. Belter, Jacob L. Segil, Aaron M. Dollar, and Richard F. Weir, Mechanical design and performance specifications of anthropomorphic prosthetic hands: A review. Journal of Rehabilitation Research and Development, vol. 50, no. 5, pp. 599-618, November 2013.

[13] Kevin O'Toole, A Methodology Towards Comprehensive Evaluation of Shape Memory Alloy Actuators for Prosthetic Finger Design. Dublin Institute of Technology, 2011.

[14] Myofilament Structure. Muscle Physiology, University of California San Diego, January 2006.

[15] Les amputés en chiffres. . Collège français des enseignants universitaires de médecine physique et de réadaptation, 2006.

[16] Paulo Silva Lobo, João Almeida, and Luís Guerreiro, Shape memory alloys behaviour: A review. 1st International Conference on Structural Integrity, no. 114, pp. 776-783, 2015.

[17] Rig Veda. Ancient Vedas, Hindu Scriptures and Texts, no. 1.118.17, 2014.

[18] Robert Vinet, Yves Lozac'h, Nicolas Beaudry, and Gilbert Drouin, Design Methodology for a Multifunctional Hand Prosthesis. Journal of Rehabilitation Research and Development, vol. 32, no. 4, pp. 316-324, November 1995.

[19] Technical Characteristics of Flexinol Actuation Wires. Dynalloy, Inc., Makers of Dynamic Alloys, 2017. 